\title{
Emotional intelligence, social emotions, parental involvement in the emotional life of senior preschoolers: development and interrelation
}

\author{
Olga Lebedenko*
}

Southern Federal University, 344006 Rostov-on-Don, Russia

\begin{abstract}
The article analyzes the category of emotional intelligence and gives grounds for its significance in the social situation of the development of preschoolers. Various approaches to the analysis of the issue of emotional intelligence of a person are presented, the characteristics of its formation in preschool age are shown. Partial programs on the development of the emotional sphere and emotional intelligence of preschoolers are briefly reviewed. Empirically it has been proved that there is an interrelation between the degree of differentiation of social emotions of children with the level of emotional intelligence of preschoolers, as well as an interrelation between the indicators of emotional intelligence of senior preschoolers and the degree of parental involvement in the emotional life of their children. The necessity of organizing work on the development of emotional intelligence of senior preschoolers is substantiated, provided that the children's parents are included as participants of educational relations.
\end{abstract}

\section{Introduction}

At the present stage of development of psychological science, emotional intelligence is considered as an important component of the personality, manifested in effective interaction with others. The importance of the emotional sphere of children and, in particular, the development of the emotional intelligence of preschoolers is emphasized in the fundamental regulatory documents governing the work of the educational psychologist in the field of education. Thus, the Federal Law "On Education of the Russian Federation" establishes (among other things) that the content of education should promote mutual understanding and cooperation between people, the formation and development of a human personality in accordance with spiritual, moral and sociocultural values accepted in the family and society [1]. Facilitation in prevention of violations in the emotional sphere, development and adjustment of the emotional sphere of children in order to form the personality of a preschooler as a conscious subject of behavior and social action is assumed in the professional standard of an educational psychologist, in view of the labor function of psychological prevention. [2]. In the Federal State Educational Standard for Preschool

\footnotetext{
${ }^{*}$ Corresponding author: olgaleb08@mail.ru
} 
Education, one of the priorities is to ensure the emotional well-being of students. The development of emotional intelligence is part of the social and communicative development as an educational field of the Federal State Educational Standard for Preschool Education and, as a social and psychological characteristic of a child's personality at the stage of completion of preschool education, and it assumes the ability to negotiate with peers, empathize with failures and take joy for the successes of other people. [3].

\section{Statement of the research problem}

The formation of the category of "emotional intelligence" dates back to the times of ancient psychology and has its own history, from a clear separation of emotions and reason up to the understanding of the integrity of all mental processes and their mutual influence. In Western psychology, the concept of emotional intelligence appeared thanks to American researchers John Mayer and Peter Salovey. By this term they defined the human ability to perceive emotions, their analysis, understanding, inclusion in existing knowledge about the emotional sphere, as well as the expression of emotions. [4] The category of emotional intelligence was further developed in connection with the identification by the American psychologist D. Goleman of five basic skills of emotional intelligence: knowledge and recognition of emotions; emotions management; self-motivation, that is, the use of emotions to achieve your goal; empathy (recognition of the emotions of others); relationship management with others. [5]

In the scientific works of Russian psychologists, there were some ideas expressed that contributed to the formation of the concept of "emotional intelligence". Thus, L.S. Vygotsky emphasized the dynamic connection of thinking and emotions, the idea that each stage of thinking development corresponds to a stage in the emotions development, and there is an interrelation of emotions with stable forms of person's behavior. [6] A.V. Zaporozhets spoke about the importance of studying the origin of emotions for upbringing, since the main task of upbringing is the development in children of a certain emotional attitude towards people and the surrounding reality, and not just the formation of knowledge, competence and skills. His studies of the genesis of emotions in preschool age revealed the interrelation of the emotional development of children with the content and structure of children's activity, with the characteristics of the relationship of the child with the people around him and with the success of the acquisition of rules and values accepted in society. [7]

Modern researcher I.N. Andreeva considers emotional intelligence to be an intellectual ability to process emotional information, which as a mental ability is part of a wider group of personality traits. In her studies, she came to the conclusion that emotional intelligence has close ties with general intelligence, with the ability to analyze problems, with the ability to display leadership and organizational qualities and other personality traits. People with a sufficient level of emotional intelligence are prone to prosocial behavior, fidelity, and successful family relationships. In contrast, a low level of emotional intelligence can lead a person to various types of addictions, poor interpersonal relationships, etc. [8] In the model of emotional intelligence proposed by D.V. Lyusin, emotional intelligence is defined as "a set of abilities to understand and control one's own and others' emotions." The ability to understand emotions is based on the ability to recognize and identify emotions, as well as understanding the cause and effect of the experienced emotion. [9]

However, many Russian psychologists of the past and present centuries emphasize the importance of developing emotions in preschool age as a prerequisite for the formation of the emotional intelligence of a person. A.D. Kosheleva notes in her studies that the emotional sphere of a child has a significant impact on his psyche and health in general, since it regulates a complex system [10]. E.I. Izotova and E.V. Nikiforova, speaking about the patterns of development of emotions in childhood, describe that, starting from preschool age, the modal range of emotional reactions is expanding in connection with the 
socialization of the whole process of child development. Psychologists note the emergence of "intellectual emotions" in connection with the integration of the emotional and intellectual spheres. Preschoolers undergo the process of formation of social emotions (emotional processes that are affected by social standards). While developing, social emotions regulate human activity, influencing and regulating contacts with society, eventually becoming the main mechanism of socialization. In their opinion, in senior preschool age arises one of the central new formations of development: "emotional correction of behavior", due to which emotional regulation becomes accessible to the child, which is manifested in the coordination of his behavior with a certain situation and the actions that the child performs. All this allows the child to adapt to the environment. [11] Emotional instability, anxiety or aggressiveness in a child's behavior can not only complicate his communication with the world around, but also lead to a deformation of the personal development of preschoolers in general. Therefore, in modern studies of the emotional intelligence of preschoolers, the need for organizing targeted psychological and pedagogical work focused on developing emotional intelligence and enriching the emotional experience of preschoolers is substantiated [12, 13, 14].

As part of our study, we analyzed partial programs for the development of the emotional sphere and emotional intelligence of preschoolers as a direction of social and communicative development of children in a preschool educational organization. So, for example, the main feature of the children's emotional development program "I am surprised, angry, afraid, excited and I boast" is the continuity of two age periods important for emotional development - preschool and primary school age. The authors of the program aimed at creating the conditions for introducing a child into the complex world of human emotions, helping to experience a certain emotional state, explaining and highlighting it verbally. The advantage of the program is the introduction of preschoolers to the selfregulation skills that contribute to the development of the ability to manage their emotions. The content of the partial program is implemented using the methods of fairy tales, psychodrama, blotography, the use of games and exercises on development of skills for interacting with peers and cognitive games [15].

In the last decade, programs have appeared that are aimed directly at developing the emotional intelligence of preschoolers. The author Min An Nguyen designed a program for the development of emotional intelligence in senior preschoolers. The concept of the program is based on the development of emotional intelligence as the orientation of the child on the role and significance of other people in the process of his activities. The strategic objectives of the program are: the development of children's interest in their own emotional experiences and experiences of other people; expansion of ideas about basic emotions and feelings of a person; mastering the practical ability to interact with peers. A distinctive feature of the program is the structure of group lessons with preschoolers proposed by the authors, which includes the required components: reading and analysis of literary works from the point of view of the presence of characters' emotions; work with subject and narrative pictures on the analysis of the emotional states of characters in literary works; playing practical situations in accordance with the social experience of children [16].

We can conclude that the considered programs of emotional development of preschoolers are aimed at working with children and do not imply the inclusion of other participants of educational relations in this process. However, in our opinion, an important role in the development of emotional intelligence and emotional comfort of children is played by parents, their involvement in the process of forming the knowledge about emotions and ways to control their emotional state.

\section{The goals and objectives of the study}

Given the relevance of the issue of developing the emotional intelligence of preschoolers, the goal of our empirical study was to study the interrelation between the degree of 
differentiation of social emotions of senior preschoolers and the level of emotional intelligence, taking into account the degree of parental involvement in the emotional life of the child. In accordance with the goal, the following study objectives were formulated: a) to determine the degree of differentiation of social emotions of senior preschoolers; $b$ ) to identify the level and indicators of emotional intelligence of preschoolers; c) to identify the presence of an interrelation of indicators of emotional intelligence and differentiation of social emotions of senior preschoolers; d) to identify the interrelation of indicators of emotional intelligence of senior preschoolers and parental involvement in the emotional life of the child; e) development of a program of psychological and pedagogical support for the development of emotional intelligence of senior preschoolers. The study involved senior preschoolers (5 - 7 years old) attending the Municipal Autonomous Educational Institution "School No. 96 Eureka-Development" in Rostov-on-Don. The total sample of respondents was 81 people.

\section{Study methods}

"Houses" method (author - O.A. Orekhova), "Diagnosis of the level of development of the emotional intelligence of a senior preschooler" method (author - Min An Nguyen), "Representations of parents about the emotional characteristics of child" questionnaire (author - E.I. Izotova), mathematical statistics methods (Spearman correlation analysis).

\section{Study results}

The first stage of the empirical study was to study the characteristics of the emotions of the social genesis of senior preschoolers using the "Houses" methodology (O.A. Orekhova). This method allows you to analyze the emotional sphere of a child according to three parameters: to assess the degree of differentiation of emotions (positive feelings are painted in bright colors, and negative ones are painted in dark colors); to reveal the presence of ambivalence (inability to distinguish between positive and negative emotions); determine the presence of inversions (when a negative emotion causes pleasure in a child). Qualitative and quantitative analysis of the results of the study allowed us to identify four groups of preschoolers. The first group of respondents was children (34\%), who correctly differentiated emotions, that is, those who preferred positive and rejected negative emotions. Such children have a high level of mental health resources, which provides good adaptation in life situations. The second group included children with a high level of mental health resources $(5 \%)$, but not distinguishing some opposing emotions. It can be assumed that emotional issues can deepen in such children when under stress. The third group of respondents $(48 \%)$ - with an inverted emotional sphere, with a predominance of negative and rejection of positive emotions - was the largest. That is, preschoolers differentiate emotions, but this differentiation is of the inverted character. The fourth group of subjects $(13 \%)$ included preschoolers with both ambivalence and inversion of emotions. Such children have low emotional resources and for this reason may have physical and mental health problems. In general, we can talk about the low level of development of the emotional sphere of respondents and problems in the differentiation of social emotions by the end of preschool age.

At the second stage of the study, the level of development of the emotional intelligence of senior preschoolers was diagnosed with the help of a set of projective techniques by M.A. Nguyen: "Finishing drawing: world of things - world of people - world of emotions" method, aimed at identifying the emotional orientation of the child to the world of things or to the world of people; "Three Wishes" method, revealing the child's emotional orientation to oneself or to other people; "What - why - how" method, which determines the degree of the child's readiness to take into account the emotional state of another person, empathize, take care of him. An analysis of the results of the study of emotional intelligence showed 
that half of the respondents have a medium level of emotional intelligence $(51 \%)$, preschoolers with a high and low level of emotional intelligence $(25 \%$ and $24 \%$, respectively) are in equal proportion. An analysis of emotional intelligence in accordance with the criteria confirms the overall diagnostic results: half of the respondents $(53 \%)$ have a medium level according to the criterion "orientation to the world of people"; $27 \%$ of preschoolers have a high level of orientation to the world of people, and only $20 \%$ of children have a low level of orientation to the world of people. According to the criterion "emotional orientation of the child towards oneself or other people", most preschoolers $(70 \%)$ have a low indicator of orientation to other people, the medium and high indicator of orientation to other people are in the same ratio $(16 \%$ and $14 \%$ of respondents, respectively). According to the criterion "degree of readiness of the child to take into account the emotional state of another person, empathize, take care of him", it turned out that the majority of respondents have a medium level of ability to empathize $(63 \%)$, the next largest group was a group of children with a low level of understanding the emotional state of another person and empathize (27\%), and only a small part of preschoolers have a high level of willingness to understand the emotional state of another person (10\%).

At the third stage, the degree of parental involvement in the emotional life of the child was studied using the questionnaire "Parents' representations about the emotional characteristics of the child" (author - E.I. Izotova). A high degree of parental involvement in the emotional life of children was identified among $41 \%$ of parents, a medium degree among $44 \%$ and a low degree among $15 \%$ of parents. At the same time, situations of emotional rejection of the child were not identified.

The fourth stage of the study was to identify the interrelation of the studied variables. Using Spearman correlation analysis, significant positive correlation relations were identified. Indicators of emotional intelligence are positively interrelated with indicators of differentiation of social emotions of senior preschoolers $(p=0.00001)$. The greater the degree of differentiation of social emotions, the higher the level of development of the emotional intelligence of senior preschoolers. Indicators of emotional intelligence of senior preschoolers are positively correlated with indicators of parental involvement in the emotional life of children $(\mathrm{p}=0.001)$. The data obtained prove that the level of emotional intelligence of preschoolers depends on the degree of parental involvement in the emotional life of children.

The results of an empirical study convincingly prove the need to organize the activity of educational psychologist to support the development of emotional intelligence of preschoolers. We have designed a program of psychological and pedagogical support for the development of emotional intelligence of senior preschoolers. The program structure has three main blocks. The diagnostic block of the program includes the study of the characteristics of the emotional intelligence of children and the level of awareness of parents on this issue. The developing block of the program is represented by a system of classes with senior preschoolers focused on development of the perception of their feelings and feelings of other people, development of self-esteem and self-awareness, development of social contact skills. The educational block of the program is aimed at improving the literacy and psychological and pedagogical competence of parents and teachers on the issues of the development of the emotional sphere of children. The content of the program is implemented by educational psychologist using effective forms of working with children (psycho-gymnastics, theatrical games, simulation games, art therapy exercises, etc.); with parents (training sessions, educational seminars, analysis of problem pedagogical situations); with teachers (master classes, business games, trainings). The main form of organizing the educational process is group lessons, however, individual counseling of participants of the program of psychological and pedagogical support is also provided. Thus, the organization of purposeful and systematic work of all participants of educational relations will contribute to the awareness of social emotions and the development of 
emotional intelligence components by senior preschoolers in combination with the active participation of parents in the emotional life of the child.

\section{Findings}

Spearman's correlation analysis led to the following conclusions: interrelation between the degree of differentiation of social emotions of children and the level of emotional intelligence of senior preschoolers is identified; interrelation between the indicators of emotional intelligence of senior preschoolers and the degree of parental involvement in the emotional life of children is identified; organization of work on the development of emotional intelligence of senior preschoolers will be effective provided that the parents of preschoolers are involved in this process as participants of educational relationships.

\section{Conclusion}

The presented program of psychological and pedagogical support for the development of emotional intelligence of senior preschoolers can act as a model for educational psychologists to create similar programs in educational organizations of various types, taking into account the characteristics of the students (primary school students, children with special needs, orphans and etc.).

\section{References}

1. Federal Law "On Education in the Russian Federation" (Moscow, 2012)

2. On the approval of the professional standard "educational psychologist" (psychologist in the field of education)" (Moscow, 2015)

3. Methodological recommendations for preschool educational organizations on the preparation of the main educational program of preschool education on the basis of the Federal State Educational Standard of Preschool Education and exemplary Basic Educational Program of Preschool Education (FIRO, 2015)

4. J.D. Mayer, P. Salovey, Intelligence. 772-781 (1993)

5. D. Goleman, Emotional leadership: The art of managing people based on emotional intelligence (Alpina Publisher, Moscow, 2012)

6. L.S. Vygotsky Collected Works: in 6 vols. 6 (Pedagogy, Moscow, 1984)

7. The development of social emotions in preschoolers: Psychological research (Pedagogy, Moscow, 1986)

8. I.N. Andreeva, Emotional intelligence as a phenomenon of modern psychology. (PSU, Novopolotsk, 2011)

9. D.V. Lyusin, Social intelligence: theory, measurement, research. (IP RAS, Moscow, 2004).

10. A.D. Kosheleva, V.I. Pereguda., O.A. Shagraeva, The emotional development of preschoolers (Academy, Moscow, 2003)

11. E.I. Izotova, E.V. Nikiforova, The emotional sphere of the child. Theory and practice. (Academy, Moscow, 2004)

12. A.J. Buyanova, E.J. Blednova, Education and upbringing. 3 (2015)

13. O.A. Lebedenko, A.K. Kovalenko, Personality in culture and education: psychological support, development, socialization. 5 (2017)

14. E.A. Azarova, G.N. Zhulina, E.G. Shevyreva, Procedia - Social and Behavioral Sciences. 233 (2016)

15. S.V. Kryukova, N.P. Slobodyanik I am surprised, angry, afraid, excited and I boast. (Genesis, Moscow 2005)

16. M. A.Nguyen, Child in kindergarten. 5 (2007) 